\title{
Hitting the sweet spot
}

David A. Tirrell

\section{By taking advantage of the cell's carbohydrate metabolism, reactive sugar analogues can be used to tag specific cells, potentially singling them out for imaging studies or drug delivery.}

$\mathrm{D}$ elivering chemical agents to specific locations in the body has been an elusive goal ever since Paul Erhlich described his concept of the 'magic bullet' nearly a century ago. Whether the objective is delivering drugs to sites of injury and disease, or the infusion of imaging agents that allow physicians to see what is going on, one would like to be able to target molecules to the places where they are needed most. Ideally, one would be able to pick out a single cell (such as a cancer cell) in the midst of many others, and deliver a molecular payload to that cell alone.

Given the subtlety of the differences between cells, it is not surprising that our bullets are somewhat less magical than we would like. To be sure, there have been successes in targeting using antibodies ${ }^{1}$ and ligands for cell-surface receptors ${ }^{2}$; nevertheless, the specificity of such targeting methods remains modest - and problematic with regard to practical application.

On page 873 of this issue, Bertozzi and colleagues $^{3}$ describe a new approach for differentiating one cell from another. First, mice are treated with a reactive sugar analogue that makes its way to cell surfaces by one of the normal pathways of carbohydrate metabolism. Cells that metabolize the analogue are thereby labelled with chemical groups that do not normally appear on their surface. Next, the mice are fed with a chemical agent that reacts specifically with the newly arrived sugar labels and leaves unmarked cells alone ${ }^{4}$ (Fig. 1).

The fact that this works is remarkable. Success requires that the sugar analogue finds its way to the cell surface through the appropriate biosynthetic pathway, that the reagent delivered in the second step is selective enough to react only with labelled cells, and that the reagent forms a new chemical bond in situ. Bertozzi and colleagues show convincingly that each of these requirements can be met; labelling is readily detected in mice treated with both reagents, but not in those treated with either reagent alone. There is also evidence that the level of labelling varies from one organ to another: in preliminary experiments, heart, liver and kidney were labelled more heavily than brain or thymus tissue, although it is not yet clear why this is so.

What might be done with this new approach? The most obvious opportunities are in fundamental studies of cell-surface
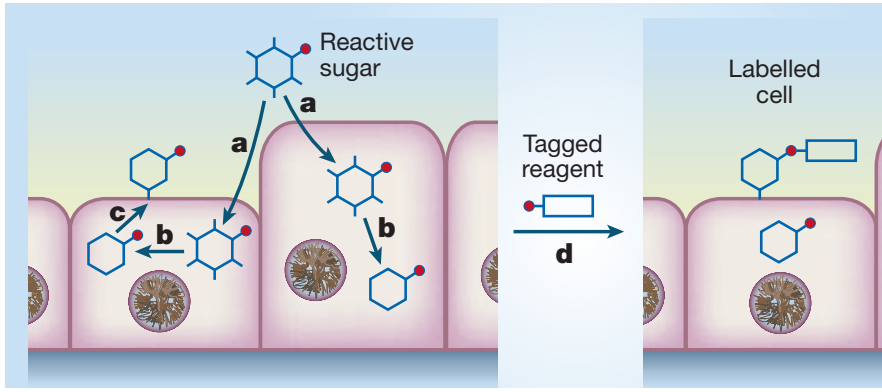

Unlabelled cell

Figure 1 Chemical labelling of cell surfaces in live mice. Bertozzi and colleagues ${ }^{3}$ report an innovative approach to targeting chemical agents to specific locations in the body. First, the mice are treated with modified sugars, which are taken up by cells (a), undergo metabolic transformation (b) and are displayed on the cell surface (c). Next, a tagged reagent reacts specifically with the modified cell surfaces $(\mathrm{d})$. Cells that do not display the modified sugars are not labelled.

sugars. The pattern of sugars on the cell surface is crucial in cell-cell communication, in interaction with pathogens, and in mediating inflammation and disease ${ }^{5}$. The labelling strategy described by Bertozzi and colleagues allows one to probe the set of sugars arrayed by the cell, to explore biosynthetic pathways and to examine the functional consequences of modifying the complement of cell-surface sugars. The authors suggest, for example, that the relatively high levels of labelling observed in heart and kidney might be a consequence of the fact that these organs are deficient in one of the enzymes involved in the synthesis of the natural sugar that competes with the labelling analogue.

This example also demonstrates how exceptional this approach to the labelling and targeting of cells is. Ordinarily, a cellular target is selected because it is a molecule that is already there - perhaps an antigen that appears on one type of cell and not on another ${ }^{1}$. But in the Bertozzi approach, targeting also depends on factors (such as a deficiency or surfeit of a biosynthetic enzyme) that determine the extent to which the surface can be decorated with reactive sugars. This opens up new strategies for discriminating between cellular targets - strategies that depend not just on structure or composition but also on function and metabolic state. A cell can be targeted not only because of what it looks like, but also because of what it is doing. Whether one is interested in imaging or in therapy, the ability to accomplish function-based targeting is likely to be an important asset ${ }^{6}$.

Insights into how cellular physiology changes over time can also be extracted from these experiments. Because cell-surface carbohydrates are made continuously, it is normally very difficult to distinguish newly synthesized variants from the large background of similar species already decorating the cell surface. But here, one labels only the newly synthesized variants, and if the chemical tag is designed properly one can use it to purify (or at least enrich) those variants for careful structural analysis. The challenges involved in such analyses are substantial but not insurmountable.

Finally, irrespective of its biological relevance, the method introduced by Bertozzi and colleagues is remarkable as a chemical process. As synthetic chemistry has advanced over the decades, the complexity of its targets and the specificity of its methods have developed in concert. From small molecules to large molecules, to molecular assemblies, materials and cells, chemists have tackled successively larger and more complex synthetic challenges ${ }^{7}$. The fact that specific chemical transformations can now be accomplished with spatial and temporal control in live animals is a major step forward for chemistry.

David A. Tirrell is in the Division of Chemistry and Chemical Engineering, California Institute of Technology, Pasadena, California 91125, USA.

e-mail: tirrell@caltech.edu

1. Guillemard, V. \& Saragovi, H. U. Curr. Cancer Drug Targets 4, 313-326 (2004).

2. Lu, Y. J., Sega, E., Leamon, C. P. \& Low, P. S. Adv. Drug Delivery Rev. 56, 1161-1176 (2004).

3. Prescher, J. A., Dube, D. H. \& Bertozzi, C. R. Nature 430, 873-877 (2004).

4. Saxon, E. \& Bertozzi, C. R. Science 287, 2007-2010 (2000).

5. Lowe, J. B. \& Marth, J. D. Annu. Rev. Biochem. 72, 643-691 (2003).

6. Li, W.-H., Fraser, S. E. \& Meade, T. J. J. Am. Chem. Soc. 121, 1413-1414 (1999).

7. Ruben, M. et al. Angew. Chem. Int. Edn 43, 3644-3662 (2004). 